\title{
Hydrodynamic excitation spectrum and time correlation functions for the multicomponent mixtures of magnetic and nonmagnetic particles
}

\author{
O.F.Batsevych ${ }^{1}$, I.M.Mryglod ${ }^{2}$, Yu.K.Rudavskii ${ }^{1}$, \\ M.V.Tokarchuk ${ }^{2}$ \\ 1 State University "Lvivska Politekhnika", \\ 12 Bandera Str., 79013 Lviv, Ukraine \\ 2 Institute for Condensed Matter Physics \\ of the National Academy of Sciences of Ukraine, \\ 1 Svientsitskii Str., 79011 Lviv, Ukraine
}

Received August 23, 2000

\begin{abstract}
The hydrodynamics of a multicomponent mixture of magnetic and nonmagnetic particles is investigated. The hydrodynamic modes spectrum is obtained. It is shown that two collective modes are responsible for the propagation of sound while the other ones $(j-2$ modes, where $j$ is determined by the number of additive integrals of motion) are purely diffusive.

The analytical expressions for all the hydrodynamic time correlation functions are derived and written via the well-defined thermodynamical quantities and transport coefficients of the system. Based on this, the expressions for the dynamical structure factors for a binary magnetic mixture in the paramagnetic state and for a three-component nonmagnetic mixture are derived.
\end{abstract}

Key words: multicomponent magnetic mixtures, time correlation functions

PACS: $75.50 . \mathrm{Mm}, 05.60 .+w, 51.10 .+y, 05.70 . \mathrm{Ln}, 05.20 .-y$

\section{Introduction}

Magnetic liquids, mixtures of magnetic and nonmagnetic particles in the external fields of mechanical or electromagnetic origin, have already taken their significant place in chemical, electronic and other modern technologies. The recent experimental investigations [1] have already proved the existence of a ferromagnetic phase in a supercooled liquid alloy $\mathrm{Co}_{80}-\mathrm{Pd}_{20}$. Similar results were obtained previously for an alloy $\mathrm{Au}-\mathrm{Co}[2-4]$ with Heisenberg exchange interaction. Description of spin relaxation in liquids [5], investigations of the dynamics of ferrocolloid systems [6-8], 
dynamical properties of polar liquids [9-11], surface absorption dynamics [11,12], etc, still need rigorous theoretical explanation.

The statistical hydrodynamics for a mixture of magnetic and non-magnetic particles in a non-homogeneous external magnetic field has been presented in $[13,14]$. Using the method of non-equilibrium statistical operator $[15,16]$, the generalized hydrodynamic equations, valid for describing both strong and weak non-equilibrium states, are derived herein. To study weak non-equilibrium processes, the linearized equations of molecular hydrodynamics and the equations for time correlations functions were found, and corresponding memory functions were analyzed.

The goal of this paper is to investigate the hydrodynamic region of wave number $k$ and frequency $\omega$, using the results obtained in $[13,14]$. The peculiarity of the proposed approach is that the results are presented for a more general case of a multicomponent mixture which includes an arbitrary number of magnetic and nonmagnetic components. In section 3 the hydrodynamic modes spectrum is found. Time dependent correlation functions are analyzed in section 4, where the analytical expressions for weight coefficients describing contributions of each mode to time correlation functions are given. The subsequent sections are devoted to the analysis of particular cases of mixtures. In sections 6 and 7, the cases of binary magnetic mixture and three-component nonmagnetic mixture are analyzed. The hydrodynamic modes spectrum, expressions for dynamical structure factors and magnetic structure factor (for a binary magnetic mixture) are written via the thermodynamical parameters and transport coefficients.

\section{Equations of molecular hydrodynamics}

Let us consider a general case of multicomponent liquid mixture in hydrodynamic limit with an arbitrary number of magnetic and nonmagnetic components [17-19]. The results obtained will be applied for particular cases of simple, binary ferromagnetic liquid, three-component mixture of simple fluids. While studying the dynamics of such a generalized multicomponent magnetic mixture (GMMM), we will use the method of nonequilibrium statistical operator $[15,16]$, so that an appropriate set of parameters of abbreviated description (also called "relevant variables") has to be chosen. These parameters, averages of which are expected to describe the dynamics of the system most adequately, should include the densities of all the conserved quantities. Considering an $m_{1}$-component magnetic mixture $\left(m_{1}\right.$ is number of both magnetic and nonmagnetic species), we introduce the first portion of the relevant parameters as the Fourier-transforms of microscopical densities of particle numbers $\left\{\hat{n}_{1}(k), \hat{n}_{2}(k), \ldots, \hat{n}_{m_{1}}(k)\right\}$, where $\hat{n}_{i}(k)=\sum_{j=1}^{N_{i}} \mathrm{e}^{\mathrm{i} \boldsymbol{k} \boldsymbol{r}_{j}^{(i)}}$. The next two parameters are the densities of total momentum $\hat{p}(k)$ and the enthalpy $\hat{h}(k)$ which is related to the energy density [17]. The last portion of the parameters of the abbreviated description which correspond to the spin subsystem, can be chosen as densities of partial magnetizations $\hat{s}_{1}(k), \ldots, \hat{s}_{m_{2}}(k)$, where $m_{2}$ is the number of magnetic components, if the operators of partial magnetizations $\hat{S}_{i}\left(=\hat{s}_{i}(k=0)\right)$ commute with the Hamiltonian of the system considered. Otherwise, the parameters of the abbre- 
viated description should be chosen as some of them which are conserved (commute with Hamiltonian) when $k=0$. So, let $m_{2}$ will be the member of these variables, connected with the spin subsystem. Performing the orthogonalization procedure (see [19]) for the set of variables listed above, we get the set of $m+2$ parameters of the abbreviated description:

$$
\hat{Y}(k)=\left\{\hat{p}(k), \hat{h}(k), \hat{n}_{1}(k), \ldots, \hat{n}_{m_{1}}(k), \hat{s}_{1}(k), \ldots, \hat{s}_{m_{2}}(k)\right\},
$$

where $m=m_{1}+m_{2}$.

Considering a GMMM in the hydrodynamic state, for small deviations of the system from an equilibrium, we can write the equations of molecular hydrodynamics in the matrix form [17] as follows:

$$
\{\mathrm{i} \omega \cdot \tilde{1}-\mathrm{i} \tilde{\Omega}(k)+\tilde{\Phi}(k, \mathrm{i} \omega+\varepsilon)\}\left\langle\Delta \hat{Y}_{i}(k)\right\rangle^{\omega}=0 .
$$

The Laplace transforms of the time correlation functions, $\hat{F}(k, z)$, satisfy the equation

$$
\{z \cdot \tilde{1}-\mathrm{i} \tilde{\Omega}(k)+\tilde{\Phi}(k, z)\} \tilde{F}(k, z)=\tilde{F}_{0}(k),
$$

with $z=\mathrm{i} \omega+\varepsilon$ and $\varepsilon=+0$, where $\mathrm{i} \tilde{\Omega}(k)$ and $\tilde{\Phi}(k, z)$ are the $(m+2) \times(m+2)$ matrices of frequencies and of memory functions, respectively, defined as follows:

$$
\begin{aligned}
\mathrm{i} \tilde{\Omega}(k) & =(\mathrm{i} \hat{L} \cdot \hat{Y}(k), \hat{Y}(-k))\left(\hat{Y}(k), \hat{Y}^{+}(-k)\right)^{-1}, \\
\tilde{\Phi}(k, z) & =\left((1-\mathcal{P}) \mathrm{i} \hat{L} \cdot \hat{Y}, \frac{1}{z+(1-\mathcal{P}) \mathrm{i} \hat{L}}(1-\mathcal{P}) \mathrm{i} \hat{L} \cdot \hat{Y}^{+}\right)\left(\hat{Y}(k), \hat{Y}^{+}(-k)\right)^{-1}
\end{aligned}
$$

Here $\mathrm{i} \hat{L}$ is a Liouville operator of the system and $\mathcal{P}$ is a projection Mori operator which obeys:

$$
\mathcal{P} \cdot \tilde{A}=(\tilde{A}, \hat{Y}(-k))\left(\hat{Y}(k), \hat{Y}^{+}(-k)\right)^{-1} \hat{Y}(k),
$$

and $(\hat{A}, \hat{B})$ denotes a correlation function:

$$
(\hat{A}, \hat{B})=\int_{0}^{1}\left\langle\hat{A} \rho_{0}^{\tau} \hat{B} \rho_{0}^{-\tau}\right\rangle \mathrm{d} \tau
$$

where $\langle\ldots\rangle$ is an average with respect to the equilibrium Gibbs distribution $\rho_{0}$, and $\hat{Y}(k)$ is the parameters of the abbreviated description column vector.

The static correlations function matrix $\tilde{F}_{0}(k)=\left(\hat{Y}_{i}(k), \hat{Y}_{i}^{+}(k)\right)$ in the right-hand side of (3) for the orthogonalized parameters of the abbreviated description (1), has a quasi-diagonal structure [19].

Due to conservation of the parameters of the abbreviated description, the frequency matrix (4) in hydrodynamic approximation is linear with respect to $k$, while the matrix of the memory functions is of order $k^{2}$. Hence:

$$
\mathrm{i} \tilde{\Omega}=\mathrm{i} k \cdot \tilde{\nu}, \quad \tilde{\Phi}=(\mathrm{i} k)^{2} \cdot \tilde{\varphi}
$$


where frequency matrix $\tilde{\nu}$ and matrix of memory functions $\tilde{\varphi}$ for the special choice of the parameters of the abbreviated description (1) have the following structure [19]:

$$
\tilde{\nu}=\left(\begin{array}{cccc}
0 & \nu_{1,2} & \cdots & \nu_{1, m+2} \\
\nu_{2,1} & 0 & \cdots & 0 \\
\vdots & \vdots & \ddots & \vdots \\
\nu_{m+2,1} & 0 & \cdots & 0
\end{array}\right), \quad \tilde{\varphi}=\left(\begin{array}{cccc}
\varphi_{1,1} & 0 & \cdots & 0 \\
0 & \varphi_{2,2} & \cdots & \varphi_{2, m+2} \\
\vdots & \vdots & \ddots & \vdots \\
0 & \varphi_{m+2,2} & \cdots & \varphi_{m+2, m+2}
\end{array}\right)
$$

The index ' 1 ' in (9) corresponds to the momentum variable $\hat{p}(k)$ in the set $(1)$. We note that all the nonzero elements of matrices $\tilde{\nu}$ and $\tilde{\varphi}$ can be expressed [19] via the thermodynamical parameters and transport coefficients defined by Green-Cubo formulas, respectively.

\section{Hydrodynamic collective modes}

The hydrodynamic excitation spectrum for a GMMM can be found from the eigenvalues problem for the hydrodynamic matrix $\tilde{T}_{\mathrm{H}}=\mathrm{i} \tilde{\Omega}-\tilde{\Phi}$. As it follows from $(8)$, the value $i k$ may be considered as a small parameter. Thus, for the eigenvalues $\left\{Z_{i}\right\}$ we are looking for solutions in the form of series:

$$
Z_{i}=\mathrm{i} k \lambda_{i}+(\mathrm{i} k)^{2} \cdot D_{i}+\ldots
$$

Note that terms of $(\mathrm{i} k)^{l}$ with $l>2$ are not significant, as we are restricted to the hydrodynamic range.

The coefficients $\lambda_{i}$, which are responsible for the propagation processes in a GMMM, can be easily found as eigenvalues of matrix $\tilde{\nu}$. They are:

$$
\lambda_{+}=v_{s}, \quad \lambda_{-}=-v_{s}, \quad \lambda_{0}=0,
$$

where $\lambda_{0}$ is an $m$ times degenerated solution and $v_{s}$ is the sound velocity given by:

$$
v_{s}^{2}=\frac{1}{2} \operatorname{Sp}\left(\tilde{\nu}^{2}\right)=\frac{1}{2} \sum_{i=2}^{m+2} \nu_{1, i} \cdot \nu_{i, 1},
$$

where $\operatorname{Sp}(\ldots)$ denotes the trace of the matrix. Thus, we see that in a GMMM there exists only one propagation process connected with sound excitations. The other ones are purely diffusive when $k$ is small enough.

In the next approximation for eigenvalues $Z_{i}$ (see (10)), the damping coefficients of collective modes can be found. For the sound modes they are:

$$
\Gamma \equiv D_{+}=D_{-}=\frac{\operatorname{Sp}\left(\tilde{\varphi} \tilde{\nu}^{2}\right)}{\operatorname{Sp}\left(\tilde{\nu}^{2}\right)}=\frac{\operatorname{Sp}\left(\tilde{\varphi} \tilde{\nu}^{2}\right)}{2 v_{s}^{2}}
$$

The other damping coefficients $\left\{D_{i}\right\}$, corresponding to eigenvalue $\lambda_{0}$, can be obtained from the $m$-th order algebraic equation which reads:

$$
\left|\begin{array}{cccc}
0 & \nu_{1,2} & \cdots & \nu_{1, m+2} \\
\nu_{2,1} & \varphi_{2,2}-D & \cdots & \varphi_{2, m+2} \\
\vdots & \vdots & \ddots & \vdots \\
\nu_{m+2,1} & \varphi_{m+2,2} & \cdots & \varphi_{m+2, m+2}-D
\end{array}\right|=0
$$


Finally, we conclude that hydrodynamic modes spectrum (10) is given by two conjugated sound propagation modes

$$
Z_{ \pm}= \pm \mathrm{i} k \cdot v_{s}-(\mathrm{i} k)^{2} \cdot \Gamma
$$

and by $m$ purely diffusive modes

$$
Z_{i}=-k^{2} \cdot D_{i}, \quad i=1, \ldots, m
$$

where all coefficients are defined by equations (12), (13) and (14).

\section{Time-dependent correlation functions}

The formal solution for spectral functions $\tilde{F}(k, z)$ can be found from (3)

$$
\tilde{F}(k, z)=\{z-\mathrm{i} \tilde{\Omega}(k)+\tilde{\Phi}(k, z)\}^{-1} \tilde{F}_{0}(k)=\tilde{M}(z) \cdot \tilde{F}_{0}(k) .
$$

From the matrix theory [20] one gets for $M(z)$ the following representation

$$
M(z)=\left(z \cdot \tilde{1}-\tilde{T}_{\mathrm{H}}(k)\right)^{-1}=\sum_{i=1}^{m+2}\left(z-Z_{i}\right)^{-1} \tilde{G}^{i}
$$

where $Z_{i}$ are the eigenvalues (15), (16) of matrix $\tilde{T}_{\mathrm{H}}$ and $\tilde{G}^{i}$ are the so-called weight coefficients. The formula (18) is a particular case of a more general expression

$$
f\left(\tilde{T}_{\mathrm{H}}\right)=\sum_{i=1}^{m+2} f\left(Z_{i}\right) \tilde{G}^{i},
$$

which is valid for an arbitrary analytical function $f(x)$. This last result can be easily proved using the properties of the matrix $\tilde{T}_{\mathrm{H}}$.

The weight coefficients $\tilde{G}^{i}$ can be also presented as power series in (ik):

$$
\tilde{G}^{i}=\tilde{g}_{0}^{i}+\mathrm{i} k \cdot \tilde{g}_{1}^{i}+\ldots, \quad i=1, \ldots, m .
$$

Note that the higher order terms (starting from quadratic one) are not important for the hydrodynamic domain of $k$.

For calculations of the weight coefficients we used the representation (19) for different choices of $f(x)$. After some algebra we find:

$$
\begin{aligned}
& \tilde{g}_{0}^{ \pm}=\frac{\tilde{\nu}\left(\tilde{\nu} \pm v_{s}\right)}{2 v_{s}^{2}}, \\
& \tilde{g}_{0}^{i}=\prod_{k=1(\neq i)}^{m} \tilde{P} \frac{D_{k}-\tilde{\varphi}}{D_{k}-D_{i}} \tilde{P}, \quad i=1, \ldots, m \\
& \tilde{g}_{1}^{ \pm}= \pm \frac{1}{v_{s}} \tilde{g}_{0}^{ \pm} \tilde{\varphi}\left(\tilde{P}+\frac{1}{2} \tilde{g}_{0}^{\mp}\right) \pm \frac{1}{v_{s}}\left(\tilde{P}+\frac{1}{2} \tilde{g}_{0}^{\mp}\right) \tilde{\varphi} \tilde{g}_{0}^{ \pm}, \\
& \tilde{g}_{1}^{j}=\frac{1}{v_{s}^{2}}\left(\tilde{R}_{j} \cdot \tilde{\varphi}-1\right) \tilde{\nu} \tilde{\varphi} \tilde{g}_{0}^{j}+\frac{1}{v_{s}^{2}} \tilde{g}_{0}^{j} \tilde{\varphi} \tilde{\nu}\left(\tilde{\varphi} \cdot \tilde{R}_{j}-1\right),
\end{aligned}
$$


where

$$
\begin{aligned}
\tilde{P} & =1-\tilde{\nu}^{2} / v_{s}^{2} \\
\tilde{R}_{j} & =\sum_{k=1(\neq j)}^{m} \frac{\tilde{g}_{0}^{k}}{D_{k}-D_{j}}, \quad j=1, \ldots, m .
\end{aligned}
$$

Finally, taking into account equations (18), (20), (15), (16), we obtain spectral functions of the system considered

$$
\tilde{F}(k, z)=\left\{\sum_{+,-} \frac{\tilde{g}_{0}^{ \pm}+\mathrm{i} k \cdot \tilde{g}_{1}^{ \pm}}{z \mp \mathrm{i} k v_{s}+k^{2} \Gamma}+\sum_{i=1}^{m} \frac{\tilde{g}_{0}^{i}+\mathrm{i} k \cdot \tilde{g}_{1}^{i}}{z+k^{2} D_{i}}\right\} \cdot \tilde{F}_{0}(k) .
$$

\section{Simple fluid}

Let us now apply the results obtained above to the case of a one-component fluid, being described by three parameters of the abbreviated description $\{\hat{n}(k)$, $\hat{p}(k), \hat{h}(k)\}$. In this case, the matrices of frequencies $\tilde{\nu}$ and of the memory functions $\tilde{\varphi}$ are

$$
\tilde{\nu}=\left(\begin{array}{ccc}
0 & n / \rho & 0 \\
1 /\left(n \kappa_{T}\right) & 0 & \alpha_{p} /\left(c_{V} \cdot \kappa_{T}\right) \\
0 & T \cdot \alpha_{p} /\left(n \cdot \kappa_{T}\right) & 0
\end{array}\right), \quad \tilde{\varphi}=\left(\begin{array}{ccc}
0 & 0 & 0 \\
0 & \eta_{l} / \rho & 0 \\
0 & 0 & \lambda / c_{V}
\end{array}\right)
$$

where $n=N / V$ and $\rho=m / V$ are the concentration and the mass density, $\eta_{l}=$ $L_{p p}=\frac{4}{3} \eta+\xi$ and $\lambda=L_{h h} / T$ are a longitudinal viscosity and thermoconductivity, respectively.

For the sound velocity, sound damping coefficient and damping coefficient of the heat mode we have found the well-known results [21]:

$$
\begin{aligned}
& v_{s}^{2}=\left(\frac{\partial P}{\partial \rho}\right)_{N S}=\frac{1}{\rho} \frac{\gamma}{\kappa_{T}}, \quad \Gamma=\frac{1}{2}\left(\frac{\eta_{l}}{\rho}+\frac{\lambda(\gamma-1)}{c_{P}}\right), \\
& D_{\mathrm{H}}=\frac{\eta_{l}}{\rho}+\frac{\lambda}{c_{V}}-2 \Gamma=\frac{\lambda}{c_{P}}
\end{aligned}
$$

where all the thermodynamical quantities in (28)-(30) are defined as follows:

$$
\begin{aligned}
& \kappa_{T}=-\frac{1}{V}\left(\frac{\partial V}{\partial P}\right)_{T, N, b}, \quad \alpha_{p}=\frac{1}{V}\left(\frac{\partial V}{\partial T}\right)_{P, N, M}, \\
& c_{V}=\frac{T}{V}\left(\frac{\partial S}{\partial T}\right)_{V N}, \quad c_{P}=\frac{T}{V}\left(\frac{\partial S}{\partial T}\right)_{P N}, \\
& \gamma=c_{P} / c_{V}
\end{aligned}
$$

and are the isothermal compressibility $\kappa_{T}$, isobaric thermal expansion coefficient $\alpha_{p}$, specific heat $c_{V}$ and $c_{P}$ at constant volume, and constant pressure, respectively. 
Performing simple calculations using the equations (21)-(26) we obtain for the hydrodynamic spectral functions the results known in the literature [21]. For instance, the dynamic structure factor is given by the generalized Landau-Placek formula:

$$
\frac{S(k, \omega)}{S(k)}=\frac{1}{2 \gamma} \sum_{+,-} \frac{k^{2} \Gamma-k\left( \pm \omega / v_{s}+k\right)\left(\eta_{l} / \rho-3 \Gamma\right)}{\left(\omega \pm k v_{s}\right)^{2}+\left(\Gamma k^{2}\right)^{2}}+\frac{\gamma-1}{\gamma} \cdot \frac{k^{2} \cdot \lambda / c_{P}}{\omega^{2}+\left(k^{2} \cdot \lambda / c_{P}\right)^{2}} .
$$

\section{Paramagnetic mixture of magnetic and nonmagnetic particles}

Let us now consider a binary mixture of magnetic and non-magnetic particles with the following parameters of abbreviated description

$$
\hat{Y}(k)=\left\{\hat{p}(k), \hat{n}_{1}(k), \hat{n}_{2}(k), \hat{s}(k), \hat{h}(k)\right\} .
$$

The frequency matrix $\tilde{\nu}$ for this case is [19]

$$
\tilde{\nu}=\left(\begin{array}{ccccc}
0 & v_{1} / \kappa_{T} & v_{2} / \kappa_{T} & \pi /\left(\chi \kappa_{T}\right) & \alpha_{p} \xi /\left(c_{V} \kappa_{T}\right) \\
c_{1} / \rho & 0 & 0 & 0 & 0 \\
c_{2} / \rho & 0 & 0 & 0 & 0 \\
\pi /\left(\rho \kappa_{T}\right) & 0 & 0 & 0 & 0 \\
T \alpha_{p} \xi /\left(\rho \kappa_{T}\right) & 0 & 0 & 0 & 0
\end{array}\right)
$$

where $\xi=1+\pi^{2} /\left(\chi \cdot \kappa_{T}\right)$ and

$$
\begin{aligned}
& \chi=\frac{1}{V}\left(\frac{\partial\langle\hat{S}\rangle}{\partial h}\right)_{N T V}, \quad \pi_{p}=\frac{1}{V}\left(\frac{\partial V}{\partial h}\right)_{P N T}, \\
& c_{i}=N_{i} / V, \quad v_{i}=\left(\frac{\partial V}{\partial N_{i}}\right)_{T P b N_{\bar{\imath}}}
\end{aligned}
$$

are the magnetic susceptibility $\chi$, coefficient of magnetostriction $\pi_{p}$, partial concentration $c_{i}$ and partial molar volume per molecule in the $i$-th species, respectively. All other themodynamic parameters are the same as in (31)-(33) and $h$ is an external magnetic field.

The matrix of the memory functions has a form opposite to that of $\tilde{\nu}$ (see equation(9)) with elements which can be expressed via the transport coefficients $L_{i k}$,

$$
\varphi_{i j}=T V \sum_{k} L_{i k}\left(\tilde{F}_{0}^{-1}\right)_{k j} .
$$

For the paramagnetic case, in which we are interested, we have

$$
\nu_{p, s}=\nu_{s, p}=0, \quad \varphi_{s, \hat{Y}}=\varphi_{\hat{Y}, s}=0,
$$

for $\hat{Y} \neq \hat{s}$. 
From the expression (12) (see also [19]) it can be proved that the sound velocity is proportional to the inverse adiabatic compressibility defined in the ensemble with constant magnetization and number of particles, namely

$$
v_{s}^{2}=-\frac{V^{2}}{\bar{M}}\left(\frac{\partial P}{\partial V}\right)_{N S M}=\left(\frac{\partial P}{\partial \rho}\right)_{N S M}
$$

where $\bar{M}=N_{1} m_{m}+N_{2} m_{n}, V, \rho, P$ are a mass, volume, mass density and pressure of the system, respectively; $m_{m}$ and $m_{n}$ are the masses of particles of magnetic and nonmagnetic species.

The sound damping coefficient (13) is given by:

$$
\Gamma=\frac{1}{2}\left(\frac{1}{v_{s}^{2}} \sum_{i, j} \nu_{p, i} \varphi_{i, j} \nu_{j, p}+\varphi_{p, p}\right) .
$$

where index ' $p$ ' in $\nu_{p, i}, \nu_{j, p}$ stands for the momentum variable.

Equation (14) for the damping coefficients of the diffusive modes can be easily factorized, and we have:

$$
D_{1}=\varphi_{s, s}, \quad D_{2 ; 3}=-\frac{1}{2} b \pm \frac{1}{2} \sqrt{b^{2}-4 c}
$$

where $D_{1}, D_{2}, D_{3}$ are the spin diffusion, mutual diffusion and thermo-diffusion coefficients, respectively, and

$$
b=2 \Gamma-\operatorname{Sp} \tilde{\varphi}_{1}, \quad c=\frac{1}{v_{s}^{2}} \operatorname{det}\left(\tilde{\nu}_{1}+\tilde{\varphi}_{1}\right) .
$$

Performing some algebra, we can also calculate all the spectral functions with weight coefficients given by (21)-(26).

Taking into account the expressions for the static structure factors

$$
S_{i j} \equiv S_{n_{i}, n_{j}}=T V\left(\frac{\partial c_{i}}{\partial \mu_{j}}\right)_{T, V, b, \mu_{\bar{\jmath}}}
$$

and introducing the following notations:

$$
\begin{aligned}
& \alpha=2 \Gamma-\eta_{l} / \rho, \\
& \gamma_{k}=\sum_{l} L_{k l} \frac{v_{l}}{\kappa_{T}}+L_{k h} \frac{\alpha_{p}}{c_{V} \kappa_{T}}, \quad \gamma_{\mathrm{H}}=\sum_{l} L_{h l} \frac{v_{l}}{\kappa_{T}}+L_{h h} \frac{\alpha_{p}}{c_{V} \kappa_{T}},
\end{aligned}
$$

where small Latin indices take the values $\{1,2\}$ (or variables $\left\{\hat{n}_{1}, \hat{n}_{2}\right\}$ ) and subscript ' $h$ ' corresponds to the heat mode, we get for the $i j$-th partial dynamical structure factor the following expression:

$$
S_{i j}(\omega, k)=\frac{T V}{2 \rho v_{s}^{2}} \sum_{ \pm} \frac{k^{2} \Gamma \cdot c_{i} c_{j}-k\left(k \pm \omega / v_{s}\right)\left(c_{i} \gamma_{j}+\gamma_{i} c_{j}-c_{i} c_{j} \cdot(\alpha+\Gamma)\right)}{\left(\omega \pm k v_{s}\right)^{2}+\left(k^{2} \Gamma\right)^{2}}
$$




$$
\begin{aligned}
& +\frac{T V}{D_{3}-D_{2}} \cdot \frac{k^{2} D_{2}}{\omega^{2}+\left(k^{2} D_{2}\right)^{2}} \cdot\left\{D_{3}\left(\frac{\partial c_{i}}{\partial \mu_{j}}\right)_{T V b \mu_{\bar{\jmath}}}+\frac{\left[c_{i} \gamma_{j}+\gamma_{i} c_{j}-c_{i} c_{j} \cdot\left(\alpha+D_{3}\right)\right]}{\left(\rho v_{s}^{2}\right)}-L_{i j}\right\} \\
& +\frac{T V}{D_{2}-D_{3}} \cdot \frac{k^{2} D_{3}}{\omega^{2}+\left(k^{2} D_{3}\right)^{2}} \cdot\left\{D_{2}\left(\frac{\partial c_{i}}{\partial \mu_{j}}\right)_{T V b \mu_{\bar{\jmath}}}+\frac{\left[c_{i} \gamma_{j}+\gamma_{i} c_{j}-c_{i} c_{j} \cdot\left(\alpha+D_{2}\right)\right]}{\left(\rho v_{s}^{2}\right)}-L_{i j}\right\},
\end{aligned}
$$

where $L_{i j}$ are the mutual diffusion coefficients, $L_{i h}$ are the thermodiffusion coefficients, $\eta_{l}=L_{p p}=\frac{4}{3} \eta+\xi$ is a longitudinal viscosity, $L_{h h}$ is a coefficient of thermoconductivity. No spin contribution to $S_{n_{1}, n_{1}}(k, \omega)$ is found, as it should be expected $[22]$.

It can be easily proved that the weight coefficients $\tilde{g}_{1}^{i}$ (see (24)) for the diffusive modes will be nonzero only for the spectral functions which involve the momentum density as one of their variables. Thus, for example, from two Fourier-transforms $F_{p, p}(k, \omega)$ and $F_{p, h}(k, \omega)$ of the time correlation functions, only the second one will have a non-zero linear contributions from the diffusive modes:

$$
\begin{aligned}
F_{p, p}(k, \omega) / F_{p, p}(k)= & \frac{1}{2} \sum_{+,-} \frac{k^{2} v_{s} \Gamma \pm k\left(\omega \mp k v_{s}\right)\left(\varphi_{p, p}-2 \Gamma\right)}{\left(\omega \mp k v_{s}\right)^{2}+\left(v_{s} \Gamma k^{2}\right)^{2}}, \\
F_{p, h}(k, \omega) / F_{h, h}(k)= & \frac{1}{2} \sum_{+,-} \frac{ \pm k^{2} v_{s} \Gamma \cdot \nu_{p, h}+k\left(\omega \mp k v_{s}\right)\left(\xi_{h}^{\prime}+\nu_{p, h}\left(\varphi_{p, p}-2 \Gamma\right)\right)}{\left(\omega \mp k v_{s}\right)^{2}+\left(v_{s} \Gamma k^{2}\right)^{2}} \\
& +\sum_{\mu=2}^{3} \frac{k \omega\left(\tilde{g}_{1}^{\mu}\right)_{p, h}}{\omega^{2}+\left(v_{s} D_{\mu} k^{2}\right)^{2}},
\end{aligned}
$$

The expressions for $\left(\tilde{g}_{1}^{\mu}\right)_{p, h}$ with $\mu=2,3$ are too complicated and will be given elsewhere [23].

Because of properties (40), the spin-spin dynamical structure factor can be easily calculated and has the simple form

$$
F_{s, s}(k, \omega) / F_{s, s}=\frac{k^{2} v_{s} D_{1}}{\omega^{2}+\left(D_{1} k^{2}\right)^{2}}
$$

It is worth noticing here that the other spectral functions, constructed on the variables $\left\{n_{1}, n_{2}, p, h\right\}$, have got the expressions similar to the ones known for a binary mixture of simple liquids $[24,25]$.

\section{Three-component mixture of non-magnetic particles}

The case of a three-component non-magnetic fluid is very similar to the previous one. It is described by 5 parameters of the abbreviated description $\left\{\hat{p} ; \hat{n}_{1}, \hat{n}_{2}, \hat{n}_{3} ; \hat{h}\right\}$, which are the densities of the momentum, the partial densities of particles, and the enthalpy, respectively. Here and further all the Latin indices will take the values $\{2,3,4\}$ (or variables $\left\{n_{1}, n_{2}, n_{3}\right\}$ ). The sound damping coefficient $\Gamma$ as well as other damping coefficients $D_{i}$ for this case can be derived from the expressions (13) and 
(14). The static correlation functions $S_{i j}$ and the elements of the frequency matrix $\nu_{p, i}, \nu_{i, p}$, with $i=2,3,4$ are in fact the generalizations of what is found for the previous case (see (36), (45)). Considering the first approximations for the matrix of the weight coefficients (24), (26) we can conclude that they have the same structure as matrix $\tilde{\nu}$ and thus will give no contribution to the dynamical structure factors.

The dynamical structure factors for a three-component mixture can be written as follows:

$$
\begin{aligned}
S_{i j}(k, \omega)= & \frac{T V}{2 \rho v_{s}^{2}} \sum_{ \pm} \frac{\Gamma k^{2} \cdot c_{i} c_{j}-k\left(k \pm\left(\omega / v_{s}\right)\right)\left(c_{i} \gamma_{j}+\gamma_{i} c_{j}-(\alpha+\Gamma) c_{i} c_{j}\right)}{\left(\omega \pm k v_{s}\right)^{2}+\left(\Gamma k^{2}\right)^{2}} \\
& +T V \sum_{\mu=1}^{3} \frac{D_{\mu} k^{2} \mathcal{G}_{i j}^{\mu}}{\omega^{2}+\left(D_{\mu} k^{2}\right)^{2}}
\end{aligned}
$$

where

$$
\alpha=\frac{1}{\rho v_{s}^{2}}\left(\gamma_{k} \frac{v_{k}}{\kappa_{T}}+\gamma_{\mathrm{H}} \frac{\alpha_{p}}{c_{V} \kappa_{T}}\right), \quad \beta=\frac{1}{\rho v_{s}^{2}}\left(\gamma_{k}\left(\frac{\partial \mu_{k}}{\partial c_{l}}\right)_{N_{\bar{l}}, V, T} \gamma_{l}+\frac{\gamma_{\mathrm{H}}^{2}}{T c_{V}}\right),
$$

and $\gamma_{k}, \gamma_{\mathrm{H}}$ are given by the (47). For example, the weight coefficient $\mathcal{G}_{i j}^{1}$ with $\mu=1$ can be written as follows:

$$
\begin{aligned}
& \mathcal{G}_{i j}^{1}=\frac{1}{\left(D_{2}-D_{1}\right)\left(D_{3}-D_{1}\right)}\left\{D_{2} D_{3}\left(\frac{\partial c_{i}}{\partial \mu_{j}}\right)_{\mu_{\bar{j}}, V, T}-\left(D_{2}+D_{3}\right) L_{i j}\right. \\
& +L_{i m}\left(\frac{\partial \mu_{m}}{\partial c_{k}}\right)_{N_{\bar{k}}, V, T} L_{k j}+\frac{L_{i h} L_{h j}}{T c_{V}} \\
& \left.+\frac{\left(c_{i} \gamma_{j}+\gamma_{i} c_{j}\right)\left(\alpha+D_{2}+D_{3}\right)+c_{i} c_{j}\left[\beta-\left(\alpha+D_{2}\right)\left(\alpha+D_{3}\right)\right]-c_{i} \delta_{j}-\delta_{i} c_{j}-\gamma_{i} \gamma_{j}}{\rho v_{s}^{2}}\right\}
\end{aligned}
$$

where $\delta_{j}=L_{i l}\left(\partial \mu_{l} / \partial c_{k}\right)_{N_{\bar{k}}, V, T} \gamma_{k}+L_{i h} \gamma_{\mathrm{H}} /\left(T c_{V}\right)$. The coefficients $\mathcal{G}_{i j}^{2}$ and $\mathcal{G}_{i j}^{3}$ can be derived from (54) by cyclic permutation of diffusion coefficients $\left\{D_{1}, D_{2}, D_{3}\right\}$.

In conclusion, we note that the results obtained in this paper:

- are valid in the hydrodynamic range of wave numbers $k$ and frequencies $\omega$;

- are quite general to be applied for an arbitrary mixture of simple liquids or mixture of magnetic and nonmagnetic particles.

In the last case it is important that the spin-spin interactions should be isotropic and depend only on the distance between particles. To illustrate, we considered three different cases of model systems (simple liquid, a binary mixture of magnetic and nonmagnetic particles, and a three-component mixture of simple fluids). It was shown that the results known in the literature for a simple liquid and a binary mixture can be easily reproduced in our approach. The results found for a threecomponent mixture have been obtained for the first time and can be especially useful for the interpretation of scattering experiments. 


\section{Acknowledgements}

I.M. thanks for the support of the Fonds für Förderung der Wissenschaftlichen Forschung under project P12422 TPH.

\section{References}

1. Albrecht K., Bührer C., Fähmle M., Maier K., Platzek D., Reske J. // Appl. Phys A., 1997, vol. 65, p. 215-220.

2. Busch G., Guentherodt H. // Phys. Lett. A., 1968, vol. 27, No. 2, p. 110.

3. Kraeft B., Alexander H. // Phys. Condens. Matter, 1973, vol. 16, p. 281-287.

4. Handrich K., Kobe S. Amorphe Ferro- und Ferrimagnetica. Academica, Berlin, 1980.

5. Eds. Muus L.T. and Atkins P.W. Electron Spin Relaxation in Liquids. New York London, Plenum Press, 1972.

6. Felderhof B.U., Jones R.B. // Phys. Rev. E., 1993, vol. 48, p. 1142-1153.

7. Felderhof B.U., Jones R.B. // J. Phys. Cond. Matt., 1994, vol. 6, p. A.339-A.343

8. Rubi J.M., Miguel M.C. // Physica A., 1993, vol. 194, p. 209-217.

9. Chandra A., Wei D., Patey G.N. // J. Chem. Phys., 1993, vol. 99, p. 2068-2073.

10. Omelyan I.P., Mryglod I.M., Tokarchuk M.V. // Cond. Matt. Phys., 1998, vol. 1, p. 179-200.

11. Phase Transitions in Surface Films (eds. Tomb H., Torzo G., Lauter H.J. and Fain S.C., Jr). New York, Plenum, 1991.

12. Marx D., Nielaba P., Binder K. // Phys. Rev. B., 1993, vol. 47, p. 7788-7804.

13. Mryglod I.M., Rudavskii Yu.K., Tokarchuk M.V., Batsevych O.F. // Ukrainian J. Phys., 1999, vol. 44, No. 8 p. 1030-1039 (in Ukrainian).

14. Mryglod I.M., Rudavskii Yu.K., Tokarchuk M.V., Batsevych O.F. // Ukrainian J. Phys., 1999, vol. 44, No. 9, p. 1174-1180 (in Ukrainian).

15. Zubarev D.N. Modern methods of statistical theory of nonequilibrium processes. - In book: Itogi Nauki i Tehniki. Sovremennye Problemy Matematiki. Moskov, VINITI, 1980, vol. 15, p. 131-220 (in Russian).

16. Zubarev D.N., Morozov V., Röpke G. Statistical mechanics of nonequilibrium Processes. Vol. 1. Basic Concepts, Kinetic Theory. Berlin, Akad. Verl., 1996.

17. Mryglod I.M., Tokarchuk M.V., Folk R. // Physica A, 1995, vol. 220, p. 325-348.

18. Mryglod I.M., Folk R. // Physica A., 1996, vol. 234, p. 129-150.

19. Rudavskii Yu.K., Mryglod I.M., Tokarchuk M.V., Batsevych O.F. On the statistical hydrodynamics for a binary mixture of magnetic and nonmagnetic atoms. / Preprint of Institute for Condensed Matter Physics, ICMP-98-29E, Lviv, 1998.

20. Gantmaher F.R. The Matrix Theory. Moskov, Nauka, 1967.

21. Boon J.P., Yip S. Molecular Hydrodynamics. New York, McGraw-Hill, 1980.

22. Mryglod I.M., Folk R., Dubyk S.O., Rudavskii Yu.K. // Physica A, 2000, vol. 277, p. $389-404$.

23. Batsevych O.F., Mryglod I.M., Rudavskii Yu.K., Tokarchuk M.V. // Physica A, 2000, (in preparation).

24. Cohen C., Sutherland J.W.H., Deutch J.M. // Physics and Chemistry of Liquids, 1971, vol. 2, p. 213-235.

25. Bhatia A.B., Thornton D.E. // Physics and Chemistry of Liquids, 1974, vol. 5, p. 97111. 


\title{
Спектр гідродинамічних збуджень та часові кореляційні функції багатокомпонентних сумішей магнітних та немагнітних частинок
}

\author{
О.Ф.Бацевич ${ }^{1}$, І.М.Мриглод ${ }^{2}$, Ю.К.Рудавський ${ }^{1}$, \\ М.В.Токарчук ${ }^{2}$ \\ 1 Державний університет "Львівська Політехніка", \\ 79013 Львів, вул. С.Бандери, 12 \\ 2 Інститут фізики конденсованих систем НАН України, \\ 79011 Львів, вул. Свєнціцького, 1
}

Отримано 23 серпня 2000 р.

У роботі розглядається гідродинаміка рідкої суміші магнітних та немагнітних частинок з довільною кількістю компонент. Отримано спектр колективних збуджень і показано, що дві колективні моди $€$ звуковими, а решта $j-2$ мод є чисто дифузійними, де число $j$ визначається кількістю адитивних інтегралів руху.

Знайдено аналітичні вирази для усіх гідродинамічних часових кореляційних функцій, в яких фігурують відомі термодинамічні параметри та коефіцієнти переносу системи. На цій основі записані вирази для динамічних структурних факторів бінарної магнітної суміші у парамагнітному стані та для трикомпонентної суміші немагнітних частинок.

Ключові слова: магнітні суміші, часові кореляційні функції

PACS: $75.50 . M m, 05.60 .+w, 51.10 .+y, 05.70 . L n, 05.20 .-y$ 